\title{
Adaptive synchronization of fractional Lorenz systems using a reduced number of control signals and parameters
}

\author{
Norelys Aguila-Camacho ${ }^{\mathrm{a}, \mathrm{b}, *}$, Manuel A. Duarte-Mermoud ${ }^{\mathrm{a}, \mathrm{b}}$, Efredy Delgado-Aguilera ${ }^{\mathrm{a}, \mathrm{b}}$ \\ a Department of Electrical Engineering, University of Chile, Av. Tupper 2007, Santiago de Chile, Chile \\ ${ }^{\mathrm{b}}$ Advanced Mining Technology Center, Av. Tupper 2007, Santiago de Chile, Chile
}

\section{A R T I C L E I N F O}

\section{Article history:}

Received 4 November 2015

Revised 27 January 2016

Accepted 25 February 2016

Available online 14 March 2016

\section{Keywords:}

Minimal adaptive synchronization

Fractional Lorenz system

Synchronization

Chaotic systems

\begin{abstract}
A B S T R A C T
This paper analyzes the synchronization of two fractional Lorenz systems in two cases: the first one considering fractional Lorenz systems with unknown parameters, and the second one considering known upper bounds on some of the fractional Lorenz systems parameters. The proposed control strategies use a reduced number of control signals and control parameters, employing mild assumptions. The stability of the synchronization errors is analytically demonstrated in all cases, and the convergence to zero of the synchronization errors is analytically proved in the case when the upper bounds on some system parameters are assumed to be known. Simulation studies are presented, which allows verifying the effectiveness of the proposed control strategies.
\end{abstract}

(C) 2016 Elsevier Ltd. All rights reserved.

\section{Introduction}

The goal of the synchronization of two dynamical systems evolving separately, one called "master" and the other called "slave," is that those systems will be sharing a common trajectory from a certain time onward. The synchronization of chaotic systems has been widely studied due to its theoretical challenges and its applications in important areas such as secure communications, chemical systems, modeling brain activities [1], ecological systems [2], among others.

The synchronization can be performed under the hypothesis that system parameters are known (nonadaptive synchronization, or simply synchronization) or, if those parameters are unknown (adaptive synchronization) [3]. When the systems to be put in synchrony are described by fractional differential equations, the term fractional adaptive synchronization is used.

We can find in literature many works related to adaptive synchronization, whose results can be applied to the adaptive synchronization of fractional Lorenz systems. Different techniques have been proposed in these works, such as modified projective adaptive synchronization $[1,4,5]$, adaptive full-state linear error feedback [6-8], adaptive sliding mode control [9-12], fuzzy generalized projective synchronization [13], among others [14]. However, these techniques use the maximum possible number of control sig-

\footnotetext{
* Corresponding author. Tel.: +56229784920; fax: +56226720162.

E-mail addresses: naguila@ing.uchile.cl (N. Aguila-Camacho), mduartem@ing.uchile.cl (M.A. Duarte-Mermoud), efredy.delgado@gmail.com (E. Delgado-Aguilera).
}

nals, which in the case of the fractional Lorenz system analyzed in this work is three.

We can find some few works that can be applied to fractional Lorenz system, where only one control signal is used to make adaptive stabilization, using sliding mode control [15-17]. Applying these control techniques it is possible to stabilize a Lorenz system at the origin, using only one control signal. However, the assumption on the system structure for the application of these techniques does not allow their use in synchronization of two fractional Lorenz systems. This is because the definition of the synchronization errors lead to a structure different from the one required for the application of these techniques. Moreover, even for making stabilization of the Lorenz system using these techniques, some of the Lorenz system parameters are needed to construct the control signal, so all the system parameters can not be unknown.

In this paper we study the synchronization of two fractional Lorenz systems with unknown parameters, using a direct approach. The direct approach consists of directly adjusting the controller parameters, without identification of the unknown plant parameters. Since all the parameters of the Lorenz system are considered unknown and only one or two control signals are used to achieve synchronization, this is a work that, as far as the authors know, has not been reported in literature.

Firstly, we analyze the three possible cases where two control signals and one adjustable parameter are used. Next we analyze two cases where only one control signal and one adjustable parameter are employed. In the first four cases studied, no assumptions on the system states boundedness is made. In the fifth case, 
boundedness on the master state trajectories is assumed. The stability of the controlled systems are proved in all cases, using the fractional extension of the Lyapunov direct method, and the main difficulties in proving the convergence to zero of the synchronization errors are exposed.

An alternative solution for the five cases, where an upper bound on some of the system parameters is assumed to be known is presented as well. In these cases, not only the stability of the controlled system is proved, but the convergence to zero of the synchronization errors is proved as well, using the fractional extension of the Lyapunov direct method.

The paper is organized as follows. Section 2 presents some basic concepts of fractional calculus and stability of fractional order systems, which are used along the paper. Section 3 presents the statement of the adaptive synchronization problem, and the proposed solutions in the adaptive case. The alternative solutions assuming a known upper bound on some system parameters are presented as well. The theoretical stability analysis of the controlled system in both cases and the convergence of the synchronization errors when the upper bound on some system parameters are assumed to be known are also presented in Section 3. Section 4 presents the simulation results for the solutions proposed in Section 3, and a comparison with another control strategy available in literature. Finally, Section 5 presents the main conclusions of the work.

\section{Some concepts related to fractional calculus and stability of fractional systems}

This section presents some basic concepts of fractional calculus and stability of fractional order systems.

\subsection{Fractional calculus}

Fractional calculus studies integrals and derivatives of orders that can be any real or complex numbers [18]. The RiemannLiouville fractional integral is one of the main concepts of fractional calculus, and is presented in Definition 1.

Definition 1 (Riemann-Liouville fractional integral [18]). The Riemann-Liouville fractional integral of order $\alpha \in \mathbb{C}(\Re(\alpha)>0)$ is defined as

$I_{a+}^{\alpha} f(t)=\frac{1}{\Gamma(\alpha)} \int_{a}^{t} \frac{f(\tau)}{(t-\tau)^{1-\alpha}} d \tau$

where $t>a, \Re(\alpha)$ is the real part of $\alpha$ and $\Gamma(\alpha)$ corresponds to the Gamma Function, given by Eq. (2):

$\Gamma(\alpha)=\int_{0}^{\infty} t^{\alpha-1} e^{-t} d t$

There are some alternative definitions regarding fractional derivatives. Definition 2 corresponds to the fractional derivative according to Caputo, which is the one most frequently used in engineering problems and the one used in this paper.

Definition 2 (Caputo fractional derivative [18]). The Caputo fractional derivative of order $\alpha \in \mathbb{C}(\Re(\alpha)>0)$ is defined as

${ }_{t_{0}}^{c} D_{t}^{\alpha} x(t)=\frac{1}{\Gamma(n-\alpha)} \int_{a}^{t} \frac{f^{(n)}(\tau)}{(t-\tau)^{\alpha-n+1}} d \tau$

where $t>a, n=\Re(\alpha)+1$ for $\alpha \notin \mathbb{N}_{0} ; n=\alpha$ for $\alpha \in \mathbb{N}_{0}$.

\subsection{Stability of fractional order systems}

The known methods for stability analysis of integer order systems differ from those that have been proposed for fractional order systems. The conditions under which fractional order linear timeinvariant systems are stable were studied in [19]. However, in the case of fractional adaptive systems this analysis is not valid, since they are time-varying. The following theorem is used in this paper for the stability analysis of the adaptive fractional synchronization schemes.

Theorem 1 (Lyapunov stability and uniform stability of fractional order systems [20]). Let $x=0$ be an equilibrium point for the nonautonomous fractional-order system (4).

${ }_{t_{0}}^{C} D_{t}^{\alpha} x(t)=f(x(t), t), \quad \alpha \in(0,1)$

Let us assume that there exists a continuous function $V(x(t), t)$ such that

- $V(x(t), t)$ is positive definite.

- ${ }_{t_{0}}^{C} D_{t}^{\beta} V(x(t), t)$, with $\beta \in(0,1]$, is negative semidefinite.

then the origin of system (4) is Lyapunov stable.

- Furthermore, if $V(x(t), t)$ is decrescent,

then the origin of system (4) is Lyapunov uniformly stable.

Besides the stability and uniform stability, asymptotic stability can be proved for fractional order systems using the fractional extension of Lyapunov direct method as well, as it is stated in Theorem 2.

Definition 3. A continuous function $\gamma:[0, t) \rightarrow[0, \infty)$ is said to belong to class- $K$ if it is strictly increasing and $\gamma(0)=0$ [21].

Theorem 2 (Fractional-order extension of Lyapunov direct method [21]). Let $x=0$ be an equilibrium point for the nonautonomous fractional-order system (4). Assume that there exists a Lyapunov function $V(t, x(t))$ and class-K functions $\gamma_{i}(i=1,2,3)$ satisfying

$\gamma_{1}(\|x\|) \leq V(t, x(t)) \leq \gamma_{2}(\|x\|)$

${ }_{t_{0}}^{C} D_{t}^{\beta} V(t, x(t)) \leq-\gamma_{3}(\|x\|)$

where $\beta \in(0,1)$. Then the origin of the system (4) is asymptotically stable.

Remark 1. Given the relationship between positive definite functions and class- $K$ functions, Theorem 2 can be rewritten as in the following.

Let $x=0$ be an equilibrium point for the nonautonomous fractional-order system (4). Assume that there exists a Lyapunov function $V(t, x(t))$ positive definite and decrescent, satisfying that ${ }_{t_{0}}^{C} D_{t}^{\beta} V(t, x(t))$ is negative definite, $\beta \in(0,1)$, then the origin of system (4) is asymptotically stable.

The following lemma will be useful in proving the stability of fractional synchronization schemes, together with Theorem 1.

Lemma 1 ([20]). Let $x(t) \in \mathbb{R}^{n}$ be a vector of differentiable functions. Then, for any time instant $t \geq t_{0}$, the following relationship holds

${ }_{2}^{1}{ }^{C} D_{0} D^{\alpha}\left(x^{T}(t) P x(t)\right) \leq x^{T}(t) P_{t_{0}}^{C} D^{\alpha} x(t), \quad \forall \alpha \in(0,1]$

where $P \in \mathbb{R}^{n \times n}$ is a constant, square, symmetric and positive definite matrix.

The case when $P=I$ was treated in [22], and the specific scalar case can also be found in [23]. 


\section{Problem statement and solutions}

Let us consider the synchronization of two fractional Lorenz systems [24] formulated in the state space, one called "master system" and the other called "slave system", described by the following equations:

$\operatorname{Master}\left\{\begin{array}{l}{ }_{t_{0}}^{C} D_{t}^{\alpha} x_{m}=\sigma\left(y_{m}-x_{m}\right) \\ { }_{t_{0}}^{C} D_{t}^{\alpha} y_{m}=\gamma x_{m}-x_{m} z_{m}-y_{m} \\ { }_{t_{0}}^{C} D_{t}^{\alpha} z_{m}=x_{m} y_{m}-\beta z_{m}\end{array}\right.$

where $\alpha \in(0,1)$ and

Slave $\left\{\begin{array}{l}{ }_{t_{0}}^{c} D_{t}^{\alpha} x_{s}=\sigma\left(y_{s}-x_{s}\right)+U_{1} \\ { }_{t_{0}}^{c} D_{t}^{\alpha} y_{s}=\gamma x_{s}-x_{s} z_{s}-y_{s}+U_{2} \\ { }_{t_{0}}^{c} D_{t}^{\alpha} z_{s}=x_{s} y_{s}-\beta z_{s}+U_{3}\end{array}\right.$

where $X_{s}=\left[\begin{array}{lll}x_{m} & y_{m} & z_{m}\end{array}\right]^{T} \in \mathbb{R}^{3}$ and $X_{s}=\left[\begin{array}{lll}x_{s} & y_{s} & z_{s}\end{array}\right]^{T} \in \mathbb{R}^{3}$ are the states of the master and slave systems, respectively. $U=$ $\left[\begin{array}{lll}U_{1} & U_{2} & U_{3}\end{array}\right]^{T} \in \mathbb{R}^{3}$ is the control signal applied to the slave system, designed to achieve the synchronization of both systems. The goal is to find that $U(t)$ such that the controlled system is stable and

$\lim _{t \rightarrow \infty}\left\|X_{m}-a X_{s}\right\|=0$

i.e., to synchronize both systems except for a scaling factor $a \in \mathbb{R}$ (generalized projective synchronization [25]), which in this study is a scalar and constant factor.

It is well known that Lorenz systems [24] exhibit chaotic behavior for the following parameter values:

$\sigma=10 \quad \gamma=28 \quad \beta=8 / 3$.

We define the synchronization error as $e=\left[\begin{array}{lll}e_{1} & e_{2} & e_{3}\end{array}\right]^{T} \in \mathbb{R}^{3}$ or $e=X_{m}-a X_{s} \in \mathbb{R}^{3}$, where $a \in \mathbb{R}^{+}$is the scale factor. Then from (8) and (9), the equations describing the synchronization errors evolution are

$$
\begin{aligned}
& { }_{t_{0}}^{C} D_{t}^{\alpha} e_{1}=-\sigma e_{1}+\sigma e_{2}-a U_{1} \\
& { }_{t_{0}}^{C} D_{t}^{\alpha} e_{2}=\gamma e_{1}-e_{2}-x_{m} z_{m}+a x_{s} z_{s}-a U_{2} \\
& { }_{t_{0}}^{C} D_{t}^{\alpha} e_{3}=-\beta e_{3}+x_{m} y_{m}-a x_{s} y_{s}-a U_{3} \\
& { }^{C} \text { When } a=1 \text {, Eq. (11) turns out to be } \\
& { }_{t_{0}}^{C} D_{t}^{\alpha} e_{1}=-\sigma e_{1}+\sigma e_{2}-U_{1} \\
& { }_{t_{0}}^{C} D_{t}^{\alpha} e_{2}=\gamma e_{1}-e_{2}-x_{s} e_{3}-z_{m} e_{1}-U_{2} \\
& { }_{t_{0}}^{C} D_{t}^{\alpha} e_{3}=-\beta e_{3}+x_{m} e_{2}+y_{s} e_{1}-U_{3} .
\end{aligned}
$$

The question to be answered is how to synchronize both systems (8) and (9) to achieve and maintain a common regime as $t$ goes to infinity. Moreover, it is desired to accomplish this task without the knowledge of the parameters $\sigma, \gamma, \beta$, seeking for solutions involving a reduced number of control and states signals, as well as with a reduced number of adjustable parameters and, hopefully, without any assumption on the boundedness of the master system trajectories.

In this study we will distinguish five different cases. We will analyze first the three cases of adaptive synchronization using two control signals and one adjustable parameter, and later it is analyzed two cases using one control signal and one adjustable parameter. In the first case, we will also introduce the alternative solution using upper bounds on some of the system parameters, with the corresponding stability and convergence analysis. In the other four cases we will only introduce the alternative solution, but no stability and convergence analysis will be made for the sake of space, since the demonstration is pretty similar to the first case.

\subsection{Fractional synchronization using control signals $\mathrm{U}_{2}$ and $\mathrm{U}_{3}$}

This subsection presents the solution to the synchronization problem using control signals $U_{2}$ and $U_{3}$. The problem is addressed first in the adaptive case and later in the nonadaptive case.

Lemma 2. (Adaptive fractional synchronization using control signals. $U_{2}$ and $U_{3}$ and one adjustable parameter $\theta$ ). Let us assume that the parameters $\sigma, \gamma, \beta$ in (8) and (9) are unknown and $\sigma, \beta>0$. If the following control signals are used in (9)

$$
\begin{aligned}
U_{1} & =0 \\
U_{2} & =\frac{1}{a}\left(\theta e_{1}-x_{m} z_{m}+a x_{s} z_{s}\right) \\
U_{3} & =\frac{1}{a}\left(x_{m} y_{m}-a x_{s} y_{s}\right)
\end{aligned}
$$

where $\theta$ is an adjustable parameter with the following adaptive law

${ }_{t_{0}}^{C} D_{t}^{\alpha} \theta=\delta e_{1} e_{2}$

where $\delta$ corresponds to the adaptive gain that can be used to handle the convergence speed, then the controlled system $(11),(13),(14)$ is uniformly stable.

Proof. Using the control signals (13) in (11), the evolution of the synchronization errors results

$$
\begin{aligned}
& { }_{t_{0}}^{C} D_{t}^{\alpha} e_{1}=-\sigma e_{1}+\sigma e_{2} \\
& { }_{t_{0}}^{C} D_{t}^{\alpha} e_{2}=(\gamma-\theta) e_{1}-e_{2} \\
& { }_{t_{0}}^{C} D_{t}^{\alpha} e_{3}=-\beta e_{3}
\end{aligned}
$$

Defining the parametric error as $\phi=\gamma-\theta \in \mathbb{R}$, Eq. (15) can be expressed as

$$
\begin{aligned}
& { }_{t_{0}}^{C} D_{t}^{\alpha} e_{1}=-\sigma e_{1}+\sigma e_{2} \\
& { }_{t_{0}}^{C} D_{t}^{\alpha} e_{2}=\phi e_{1}-e_{2} \\
& { }_{t_{0}}^{C} D_{t}^{\alpha} e_{3}=-\beta e_{3}
\end{aligned}
$$

In order to prove the stability of the controlled system, let us use the fractional extension of Lyapunov direct method [20]. We propose the following Lyapunov candidate function, which is positive definite and decrescent

$V=\frac{1}{2 \sigma} e_{1}^{2}+\frac{1}{2} e_{2}^{2}+\frac{1}{2} e_{3}^{2}+\frac{1}{2 \delta} \phi^{2}$

with $\delta \in \mathbb{R}, \delta>0$

Assuming that $e_{1}, e_{2}, e_{3}, \phi$ are differentiable, then applying Lemma 1 to (17) and using (16) and (14) it can be written as

${ }_{t_{0}}^{C} D_{t}^{\alpha} V \leq-\frac{1}{2}\left(e_{1}-e_{2}\right)^{2}-\frac{1}{2} e_{1}^{2}-\frac{1}{2} e_{2}^{2}-\beta e_{3}^{2}$.

As can be seen from (18), the fractional derivative of the Lyapunov function is negative semidefinite, then it can be concluded from Theorem 1 that the origin of system (16),(14) is uniformly stable, and therefore $e_{1}, e_{2}, e_{3}, \phi \in \mathcal{L}^{\infty}$, and this concludes the proof.

Remark 2. In order to prove the convergence of the synchronization errors to zero, in the integer order case [26] it is used the Corollary of the Barbalat Lemma [27]. To this extent, besides the fact that the synchronization errors are bounded $\left(e_{1}, e_{2}, e_{3} \in \mathcal{L}^{\infty}\right)$, 
it is proved that the integer integral of the squared synchronization errors is bounded $\left(e_{1}, e_{2}, e_{3}, \phi \in \mathcal{L}^{2}\right)$ and that the errors are uniformly continuous $\left(\dot{e}_{1}, \dot{e}_{2}, \dot{e}_{3} \in \mathcal{L}^{\infty}\right)$.

However, in the fractional case it is not possible to prove that the integer integral of the squared synchronization errors is bounded, so it is no possible to use the corollary of the Barbalat Lemma [27].

Instead of this, it can be proved that the fractional integral of the squared synchronization errors is bounded (integrating expression (18)), but unfortunately there is not a fractional equivalent to the corollary of the Barbalat Lemma. That is why the analytical proof of the convergence to zero of the synchronization errors is a subject currently under research. Nevertheless, all the simulation studies accomplished during this research have shown that the synchronization errors converge to zero, as it will be shown in Section 4.

Lemma 3. (Nonadaptive fractional synchronization using control signals. $U_{2}$ and $U_{3}$, assuming a known upper bound on parameter $\gamma$ ). Let us assume that the parameters $\sigma, \gamma, \beta$ in (8) and (9) are unknown and $\gamma, \sigma, \beta>0$. It is also assumed that an upper bound $B_{\gamma}$ on the parameter $\gamma$ is known $\left(\gamma<B_{\gamma}\right)$. If the following control signals are used in (9)

$$
\begin{aligned}
U_{1} & =0 \\
U_{2} & =\frac{1}{a}\left(-x_{m} z_{m}+a x_{s} z_{s}+B_{\gamma} e_{2}\right) \\
U_{3} & =\frac{1}{a}\left(x_{m} y_{m}-a x_{s} y_{s}\right)
\end{aligned}
$$

then the controlled system (11), (19) is asymptotically stable.

Proof. Using the control signals (19) in (11), the evolution of the synchronization errors results

$$
\begin{aligned}
& { }_{t_{0}}^{C} D_{t}^{\alpha} e_{1}=-\sigma e_{1}+\sigma e_{2} \\
& { }_{t_{0}}^{C} D_{t}^{\alpha} e_{2}=\gamma e_{1}-e_{2}-B_{\gamma} e_{2} \\
& { }_{t_{0}}^{C} D_{t}^{\alpha} e_{3}=-\beta e_{3}
\end{aligned}
$$

In order to analyze the stability of the corresponding controlled system (20), let us use the fractional extension of Lyapunov direct method [21]. We propose the following Lyapunov candidate function, which is positive definite and decrescent

$V=\frac{1}{2 \sigma} e_{1}^{2}+\frac{1}{2 \gamma} e_{2}^{2}+\frac{1}{2} e_{3}^{2}$.

Note that it is the same Lyapunov function that is used in the adaptive case, except for the term including the parameter error $\left(\frac{1}{2 \delta} \phi^{2}\right)$, which in this case does not exist since this is a nonadaptive solution.

Assuming that $e_{1}, e_{2}, e_{3}$ are differentiable, applying Lemma 1 and using (20) it can be written as

$$
\begin{aligned}
{ }_{t_{0}}^{C} D_{t}^{\alpha} V & \leq-e_{1}^{2}+2 e_{1} e_{2}-\frac{1}{\gamma} e_{2}^{2}-\frac{1}{\gamma} B_{\gamma} e_{2}^{2}-\beta e_{3}^{2} \\
& \leq-\left(e_{1}-e_{2}\right)^{2}-\frac{1}{\gamma} e_{2}^{2}+e_{2}^{2}\left(1-\frac{B_{\gamma}}{\gamma}\right)-\beta e_{3}^{2} \\
& \leq-\left(e_{1}-e_{2}\right)^{2}+e_{2}^{2}\left(1-\frac{B_{\gamma}}{\gamma}\right)-\beta e_{3}^{2}
\end{aligned}
$$

Given that $\gamma<B_{\gamma}$ and $\gamma, B_{\gamma}>0$, then $\frac{B_{\gamma}}{\gamma}>1$ and consequently $\left(1-\frac{B_{\gamma}}{\gamma}\right)<0$. Using this result in expression (22), it can be concluded that the fractional derivative of the Lyapunov function is negative definite. Then using Theorem 2, it can be concluded that the origin of the system (20) is asymptotically stable, that is $e_{1}, e_{2}, e_{3} \in \mathcal{L}^{\infty}$ and

$\lim _{t \rightarrow \infty} e_{1}(t)=\lim _{t \rightarrow \infty} e_{2}(t)=\lim _{t \rightarrow \infty} e_{3}(t)=0$

and this concludes the proof.

Note that in this nonadaptive case, asymptotic stability can be proved directly from the fractional extension of Lyapunov direct method, so no additional tools are needed in order to prove that the synchronization errors converge to zero. This is due to the fact that more knowledge on the system is needed to construct the solution than in the adaptive case, since the upper bound $B_{\gamma}$ must be known.

\subsection{Fractional synchronization using control signals $\mathrm{U}_{1}$ and $\mathrm{U}_{3}$}

This subsection presents the solution to the synchronization problem using control signals $U_{1}$ and $U_{3}$. The problem is addressed in the adaptive case and in the nonadaptive case.

Lemma 4. (Adaptive fractional synchronization using control signals. $U_{1}$ and $U_{3}$ and one adjustable parameter $\theta$ ). Let us assume that the parameters $\sigma, \gamma, \beta$ in (8) and (9) are unknown, $\sigma, \beta>0$ and $a=1$. If the following control signals are used in (9)

$$
\begin{aligned}
& U_{1}=-z_{m} e_{2}+\theta e_{2} \\
& U_{2}=0 \\
& U_{3}=x_{m} e_{2}+y_{s} e_{1}-x_{s} e_{2}
\end{aligned}
$$

where $\theta$ is an adjustable parameter with the following adaptive law:

${ }_{t_{0}}^{C} D_{t}^{\alpha} \theta=\delta e_{1} e_{2}$

where $\delta$ corresponds to the adaptive gain that can be used to handle the convergence speed, then the controlled system (12), (24),(25) is uniformly stable.

Proof. Using control signals (24) in (12), the evolution of the synchronization errors turns out to be

$$
\begin{aligned}
& { }_{t_{0}}^{C} D_{t}^{\alpha} e_{1}=-\sigma e_{1}+(\sigma-\theta) e_{2}+z_{m} e_{2} \\
& { }_{t_{0}}^{C} D_{t}^{\alpha} e_{2}=\gamma e_{1}-e_{2}-x_{s} e_{3}-z_{m} e_{1} \\
& { }_{t_{0}}^{C} D_{t}^{\alpha} e_{3}=-\beta e_{3}+x_{s} e_{2}
\end{aligned}
$$

Defining the parametric error as $\phi=\sigma+\gamma-\theta$, then we can write that $(\sigma-\theta) e_{2}=\phi e_{2}-\gamma e_{2}$. Therefore, Eq. (26) can be expressed as

$$
\begin{aligned}
& { }_{t_{0}}^{C} D_{t}^{\alpha} e_{1}=-\sigma e_{1}+\phi e_{2}-\gamma e_{2}+z_{m} e_{2} \\
& { }_{t_{0}}^{C} D_{t}^{\alpha} e_{2}=\gamma e_{1}-e_{2}-x_{s} e_{3}-z_{m} e_{1} \\
& { }_{t_{0}}^{C} D_{t}^{\alpha} e_{3}=-\beta e_{3}+x_{s} e_{2}
\end{aligned}
$$

In order to prove the stability of the controlled system, we will use the fractional extension of the Lyapunov direct method [20], proposing the following Lyapunov candidate function, which 
is positive definite and decrescent:

$V=\frac{1}{2} e_{1}^{2}+\frac{1}{2} e_{2}^{2}+\frac{1}{2} e_{3}^{2}+\frac{1}{2 \delta} \phi^{2}$

with $\delta \in \mathbb{R}, \delta>0$.

Assuming that $e_{1}, e_{2}, e_{3}, \phi$ are differentiable, then applying Lemma 1 and using (27) and (25) results

${ }_{t_{0}}^{C} D_{t}^{\alpha} V \leq-\sigma e_{1}^{2}-e_{2}^{2}-\beta e_{3}^{2}$

As can be seen from (29), the fractional derivative of the Lyapunov function is negative semidefinite, then it can be concluded that the origin of system (27),(25) is uniformly stable, and $e_{1}, e_{2}, e_{3}, \phi \in \mathcal{L}^{\infty}$ and this concludes the proof.

Regarding the convergence to zero of the synchronization errors, explanation given in Remark 2 is also valid.

Lemma 5. (Nonadaptive fractional synchronization using control signals. $U_{1}$ and $U_{3}$, assuming a known upper bound on parameters $\gamma$, $\sigma)$. Let us assume that the parameters $\sigma, \gamma, \beta$ in (8) and (9) are unknown, $\beta>0$ and $a=1$. It is also assumed that an upper bound $B_{\gamma \sigma}$ on the sum $\frac{(\gamma+\sigma)^{2}}{4}$ is known $\left(\frac{(\gamma+\sigma)^{2}}{4}<B_{\gamma \sigma}\right)$. If the following control signals are used in (9)

$$
\begin{aligned}
& U_{1}=-z_{m} e_{2}+B_{\gamma \sigma} e_{1} \\
& U_{2}=0 \\
& U_{3}=x_{m} e_{2}+y_{s} e_{1}-x_{s} e_{2}
\end{aligned}
$$

then the controlled system (12),(30) is asymptotically stable.

Proof. Using the control signals (30) in (12), the evolution of the synchronization errors results

$$
\begin{aligned}
& { }_{t_{0}}^{C} D_{t}^{\alpha} e_{1}=-\sigma e_{1}+\sigma e_{2}+z_{m} e_{2}-B_{\gamma \sigma} e_{1} \\
& { }_{t_{0}}^{C} D_{t}^{\alpha} e_{2}=\gamma e_{1}-e_{2}-x_{s} e_{3}-z_{m} e_{1} \\
& { }_{t_{0}}^{C} D_{t}^{\alpha} e_{3}=-\beta e_{3}+x_{s} e_{2}
\end{aligned}
$$

For the sake of space, the stability proof is not explicitly given in this subsection. The reader can check that using the Lyapunov function candidate $V=\frac{1}{2} e_{1}^{2}+\frac{1}{2} e_{2}^{2}+\frac{1}{2} e_{3}^{2}$ and Lemma 1 , the fractional derivative of the Lyapunov function results negative definite

$$
\begin{aligned}
{ }_{t_{0}}^{C} D_{t}^{\alpha} V \leq & -\left(\frac{(\gamma+\sigma)}{2} e_{1}-e_{2}\right)^{2}-\beta e_{3}^{2} \\
& +e_{1}^{2}\left(\frac{(\gamma+\sigma)^{2}}{4}-B_{\gamma \sigma}\right)
\end{aligned}
$$

Then using Theorem 2, it can be easily concluded that the origin of system (31) is asymptotically stable, that is $\lim _{t \rightarrow \infty} e_{1}(t)=$ $\lim _{t \rightarrow \infty} e_{2}(t)=\lim _{t \rightarrow \infty} e_{3}(t)=0$.

\subsection{Fractional synchronization using control signals $\mathrm{U}_{1}$ and $\mathrm{U}_{2}$}

This subsection presents the solution to the synchronization problem using control signals $U_{1}$ and $U_{2}$. As in the previous subsections, the problem is addressed in the adaptive case and in the nonadaptive case.
Lemma 6. (Adaptive fractional synchronization using control signals. $U_{1}$ and $U_{2}$ and one adjustable parameter $\theta$ ). Let us assume that the parameters $\sigma, \gamma, \beta$ in (8) and (9) are unknown, $\sigma, \beta>0$ and $a=1$. If the following control signals are used in (9)

$$
\begin{aligned}
& U_{1}=y_{s} e_{3}+\theta e_{2} \\
& U_{2}=-x_{s} e_{3}-z_{m} e_{1}+x_{m} e_{3} \\
& U_{3}=0
\end{aligned}
$$

where $\theta$ is an adjustable parameter with the following adaptive law:

${ }_{t_{0}}^{C} D_{t}^{\alpha} \theta=\delta e_{1} e_{2}$

where $\delta$ corresponds to the adaptive gain that can be used to handle the convergence speed, then the controlled system (12),(33),(34) is uniformly stable.

Proof. Using the control signals (33) in (12), the evolution of the synchronization errors turns out to be

$$
\begin{aligned}
& { }_{t_{0}}^{C} D_{t}^{\alpha} e_{1}=-\sigma e_{1}+(\sigma-\theta) e_{2}-y_{s} e_{3} \\
& { }_{t_{0}}^{C} D_{t}^{\alpha} e_{2}=\gamma e_{1}-e_{2}-x_{m} e_{3} \\
& { }_{t_{0}}^{C} D_{t}^{\alpha} e_{3}=-\beta e_{3}+x_{m} e_{2}+y_{s} e_{1}
\end{aligned}
$$

Defining the parametric error as $\phi=\sigma+\gamma-\theta$, we can state that $(\sigma-\theta) e_{2}=\phi e_{2}-\gamma e_{2}$, and Eq. (35) can be rewritten as

$$
\begin{aligned}
& { }_{t_{0}}^{C} D_{t}^{\alpha} e_{1}=-\sigma e_{1}+\phi e_{2}-\gamma e_{2}-y_{s} e_{3} \\
& { }_{t_{0}}^{c} D_{t}^{\alpha} e_{2}=\gamma e_{1}-e_{2}-x_{m} e_{3} \\
& { }_{t_{0}}^{C} D_{t}^{\alpha} e_{3}=-\beta e_{3}+x_{m} e_{2}+y_{s} e_{1}
\end{aligned}
$$

In order to prove the stability of the controlled scheme, let us use the fractional extension of Lyapunov direct method [20], considering the following Lyapunov candidate function, which is positive definite

$V=\frac{1}{2} e_{1}^{2}+\frac{1}{2} e_{2}^{2}+\frac{1}{2} e_{3}^{2}+\frac{1}{2 \delta} \phi^{2}$

with $\delta \in \mathbb{R}, \delta>0$.

Assuming that $e_{1}, e_{2}, e_{3}, \phi$ are differentiable, then applying Lemma 1 and using (36) and (34) results

${ }_{t_{0}}^{C} D_{t}^{\alpha} V \leq-\sigma e_{1}^{2}-e_{2}^{2}-\beta e_{3}^{2}$

As can be seen from (38), the fractional derivative of the Lyapunov function is negative semidefinite, then it can be concluded that the origin of system (36),(34) is uniformly stable, that is $e_{1}, e_{2}, e_{3}, \phi \in \mathcal{L}^{\infty}$, and this concludes the proof.

Comments made in Remark 2 regarding the convergence to zero of the synchronization errors are also valid in this case.

Lemma 7. (Nonadaptive fractional synchronization using control signals. $U_{1}$ and $U_{2}$, assuming a known upper bound on parameters $\gamma$, $\sigma)$. Let us assume that the parameters $\sigma, \gamma, \beta$ in (8) and (9) are unknown, $\beta>0$ and $a=1$. It is also assumed that an upper bound $B_{\gamma \sigma}$ on the sum $\frac{(\gamma+\sigma)^{2}}{4}$ is known $\left(\frac{(\gamma+\sigma)^{2}}{4}<B_{\gamma \sigma}\right)$. If the following control signals are used in (9)

$$
\begin{aligned}
& U_{1}=y_{s} e_{3}+B_{\gamma \sigma} e_{1} \\
& U_{2}=-x_{s} e_{3}-z_{m} e_{1}+x_{m} e_{3} \\
& U_{3}=0
\end{aligned}
$$

then the controlled system (12),(39) is asymptotically stable. 
Proof. Using the control signals (39) in (12), the evolution of the synchronization errors results

$$
\begin{aligned}
& { }_{t_{0}}^{C} D_{t}^{\alpha} e_{1}=-\sigma e_{1}+\sigma e_{2}-y_{s} e_{3}-B_{\gamma \sigma} e_{1} \\
& { }_{t_{0}}^{C} D_{t}^{\alpha} e_{2}=\gamma e_{1}-e_{2}-x_{m} e_{3} \\
& { }_{t_{0}}^{C} D_{t}^{\alpha} e_{3}=-\beta e_{3}+x_{m} e_{2}+y_{s} e_{1}
\end{aligned}
$$

As in the previous case, the stability proof is not explicitly given here. The reader can easily check that using the Lyapunov function candidate $V=\frac{1}{2} e_{1}^{2}+\frac{1}{2} e_{2}^{2}+\frac{1}{2} e_{3}^{2}$ and Lemma 1 , the fractional derivative of the Lyapunov function results negative definite:

$$
\begin{aligned}
{ }_{t_{0}}^{C} D_{t}^{\alpha} V \leq & -\left(\frac{(\gamma+\sigma)}{2} e_{1}-e_{2}\right)^{2}-\beta e_{3}^{2} \\
& +e_{1}^{2}\left(\frac{(\gamma+\sigma)^{2}}{4}-B_{\gamma \sigma}\right)
\end{aligned}
$$

Then using Theorem 2, it can be proved that the origin of system $(40)$ is asymptotically stable, that is $\lim _{t \rightarrow \infty} e_{1}(t)=\lim _{t \rightarrow \infty} e_{2}(t)=$ $\lim _{t \rightarrow \infty} e_{3}(t)=0$, and this concludes the proof.

\subsection{Fractional synchronization using only control signal $\mathrm{U}_{1}$.}

This subsection presents the solution to the synchronization problem using only control signal $U_{1}$. As in the previous subsections, the problem is addressed in the adaptive case and in the nonadaptive case.

Lemma 8. (Adaptive fractional synchronization using only control signal. $U_{1}$ and one adjustable parameter $\theta$ ). Let us assume that the parameters $\sigma, \gamma, \beta$ in (8) and (9) are unknown, $\sigma, \beta>0$ and $a=1$. If the following control signals are used in (9)

$$
\begin{aligned}
& U_{1}=\theta e_{2}-y_{m} z_{s}+y_{s} z_{m} \\
& U_{2}=0 \\
& U_{3}=0
\end{aligned}
$$

where $\theta$ is an adjustable parameter with the following adaptive law:

${ }_{t_{0}}^{C} D_{t}^{\alpha} \theta=\delta e_{1} e_{2}$

where $\delta$ corresponds to the adaptive gain that can be used to handle the convergence speed, then the controlled system (12),(42),(43) is uniformly stable.

Proof. Using the control signals (42) in (12), the evolution of the synchronization errors (12) becomes

$$
\begin{aligned}
& { }_{t_{0}}^{C} D_{t}^{\alpha} e_{1}=-\sigma e_{1}+(\sigma-\theta) e_{2}+y_{m} z_{s}-y_{s} z_{m} \\
& { }_{t_{0}}^{C} D_{t}^{\alpha} e_{2}=\gamma e_{1}-e_{2}-x_{m} z_{m}+x_{s} z_{s} \\
& { }_{t_{0}}^{C} D_{t}^{\alpha} e_{3}=-\beta e_{3}+x_{m} e_{2}+y_{s} e_{1}
\end{aligned}
$$

Defining the parametric error as $\phi=\sigma+\gamma-\theta$, then Eq. (44) can be expressed as

$$
\begin{aligned}
& { }_{t_{0}}^{C} D_{t}^{\alpha} e_{1}=-\sigma e_{1}+e_{2}(\phi-\gamma)+y_{m} z_{s}-y_{s} z_{m} \\
& { }_{t_{0}}^{C} D_{t}^{\alpha} e_{2}=\gamma e_{1}-e_{2}-x_{m} z_{m}+x_{s} z_{s} \\
& { }_{t_{0}}^{C} D_{t}^{\alpha} e_{3}=-\beta e_{3}+x_{m} e_{2}+y_{s} e_{1}
\end{aligned}
$$

In order to prove the stability of the controlled system, the fractional extension of the Lyapunov direct method is used [20], proposing the following Lyapunov candidate function, which is positive definite and decrescent:

$V=\frac{1}{2} e_{1}^{2}+\frac{1}{2} e_{2}^{2}+\frac{1}{2} e_{3}^{2}+\frac{1}{2 \delta} \phi^{2}$

with $\delta \in \mathbb{R}, \delta>0$.

Assuming that $e_{1}, e_{2}, e_{3}, \phi$ are differentiable, then applying Lemma 1 and using (45) and (43) the result turns out to be

${ }_{t_{0}}^{C} D_{t}^{\alpha} V \leq-\sigma e_{1}^{2}-e_{2}^{2}-\beta e_{3}^{2}$

As can be seen from (47), the fractional derivative of the Lyapunov function is negative semidefinite, then it can be concluded that the origin of system (45),(43) is uniformly stable, that is $e_{1}, e_{2}, e_{3}, \phi \in \mathcal{L}^{\infty}$, and this concludes the proof.

Regarding the analytical proof for the convergence to zero of the synchronization errors, the comments made in Remark 2 are also valid in this case.

Lemma 9. (Nonadaptive fractional synchronization using only control signal. $U_{1}$, assuming a known upper bound on parameters $\gamma, \sigma$ ). Let us assume that the parameters $\sigma, \gamma, \beta$ in (8) and (9) are unknown, $\beta>0$ and $a=1$. It is also assumed that an upper bound $B_{\gamma \sigma}$ on the sum $\frac{(\gamma+\sigma)^{2}}{4}$ is known $\left(\frac{(\gamma+\sigma)^{2}}{4}<B_{\gamma \sigma}\right)$. If the following control signals are used in (9)

$$
\begin{aligned}
& U_{1}=-y_{m} z_{s}+y_{s} z_{m}+B_{\gamma \sigma} e_{1} \\
& U_{2}=0 \\
& U_{3}=0
\end{aligned}
$$

then the controlled system (12),(48) is asymptotically stable.

Proof. Using the control signals (48) in (12), the evolution of the synchronization errors results

$$
\begin{aligned}
& { }_{t_{0}}^{C} D_{t}^{\alpha} e_{1}=-\sigma e_{1}+\sigma e_{2}+y_{m} z_{s}-y_{s} z_{m}-B_{\gamma \sigma} e_{1} \\
& { }_{t_{0}}^{C} D_{t}^{\alpha} e_{2}=\gamma e_{1}-e_{2}+x_{s} z_{s}-x_{m} z_{m} \\
& { }_{t_{0}}^{C} D_{t}^{\alpha} e_{3}=-\beta e_{3}+x_{m} y_{m}-x_{s} y_{s} .
\end{aligned}
$$

Again in this case, for the sake of space, the detailed stability proof is not given. However, the reader can check that using the Lyapunov function candidate $V=\frac{1}{2} e_{1}^{2}+\frac{1}{2} e_{2}^{2}+\frac{1}{2} e_{3}^{2}$ and Lemma 1 , the fractional derivative of the Lyapunov function results negative definite

$$
\begin{aligned}
{ }_{t_{0}}^{C} D_{t}^{\alpha} V \leq & -\left(\frac{(\gamma+\sigma)}{2} e_{1}-e_{2}\right)^{2}-\beta e_{3}^{2} \\
& +e_{1}^{2}\left(\frac{(\gamma+\sigma)^{2}}{4}-B_{\gamma \sigma}\right)
\end{aligned}
$$

Then using Theorem 2, it can be proved that the origin of system $(49)$ is asymptotically stable, that is $\lim _{t \rightarrow \infty} e_{1}(t)=\lim _{t \rightarrow \infty} e_{2}(t)=$ $\lim _{t \rightarrow \infty} e_{3}(t)=0$ and this concludes the proof.

\subsection{Fractional synchronization using only control signal $\mathrm{U}_{2}$}

Finally, this subsection presents the solution to the synchronization problem using only control signal $U_{2}$ for both, adaptive and nonadaptive case.

Lemma 10. (Adaptive fractional synchronization using only control signal. $U_{2}$ and one adjustable parameter $\theta$ ). Let us assume that the parameters $\sigma, \gamma, \beta$ in (8) and (9) are unknown, $\sigma, \beta>0, a=1$ and that the master states trajectories $x_{m}, y_{m}$ remain bounded. If the 
following control signals are used in (9)

$$
\begin{aligned}
& U_{1}=0 \\
& U_{2}=\theta e_{1}-x_{m} z_{m}+x_{s} z_{s} \\
& U_{3}=0
\end{aligned}
$$

where $\theta$ is an adjustable parameter with the following adaptive law

${ }_{t_{0}}^{C} D_{t}^{\alpha} \theta=\delta e_{1} e_{2}$

where $\delta$ corresponds to the adaptive gain, then the controlled system (12),(51),(52) is uniformly stable.

Proof. Using the control signals (51) in (12), the evolution of the synchronization errors becomes

$$
\begin{aligned}
& { }_{t_{0}}^{C} D_{t}^{\alpha} e_{1}=-\sigma e_{1}+\sigma e_{2} \\
& { }_{t_{0}}^{C} D_{t}^{\alpha} e_{2}=(\gamma-\theta) e_{1}-e_{2} \\
& { }_{t_{0}}^{C} D_{t}^{\alpha} e_{3}=-\beta e_{3}+x_{m} e_{2}+y_{s} e_{1}
\end{aligned}
$$

Defining the parametric error as $\phi=\sigma-\theta$, thus Eq. (53) can be written as

$$
\begin{aligned}
& { }_{t_{0}}^{C} D_{t}^{\alpha} e_{1}=-\sigma e_{1}+\sigma e_{2} \\
& { }_{t_{0}}^{C} D_{t}^{\alpha} e_{2}=\phi e_{1}-e_{2} \\
& { }_{t_{0}}^{C} D_{t}^{\alpha} e_{3}=-\beta e_{3}+x_{m} e_{2}+y_{s} e_{1}
\end{aligned}
$$

In order to prove the stability of the controlled system, let us analyze first the subsystem ${ }_{t_{0}}^{C} D_{t}^{\alpha} e_{1},{ }_{t_{0}}^{C} D_{t}^{\alpha} e_{2},{ }_{t_{0}}^{C} D_{t}^{\alpha} \phi$, using the fractional extension of the Lyapunov direct method [20]. Let us consider the following Lyapunov candidate function, which is positive definite and decrescent

$V=\frac{1}{2 \sigma} e_{1}^{2}+\frac{1}{2} e_{2}^{2}+\frac{1}{2 \delta} \phi^{2}$

with $\delta \in \mathbb{R}, \delta>0$.

Assuming that $e_{1}, e_{2}, \phi$ are differentiable, then applying Lemma 1 and using (54) and (52) the result becomes

$$
\begin{aligned}
{ }_{t_{0}}^{C} D_{t}^{\alpha} V & \leq-e_{1}^{2}+e_{1} e_{2}-e_{2}^{2} \\
& \leq-\frac{1}{2}\left(e_{1}-e_{2}\right)^{2}-\frac{1}{2} e_{1}^{2}-\frac{1}{2} e_{2}^{2}
\end{aligned}
$$

As can be seen from (56), ${ }_{t_{0}}^{C} D_{t}^{\alpha} V$ is negative semidefinite, then it can be concluded that the origin of subsystem $\left({ }_{t_{0}}^{C} D_{t}^{\alpha} e_{1},{ }_{t_{0}}^{C} D_{t}^{\alpha} e_{2}\right)$ together with (52) is uniformly stable. This means that $e_{1}, e_{2}, \phi \in$ $\mathcal{L}^{\infty}$.

In order to analyze the stability of $e_{3}$, let us analyze the third equation of (54), which has the form

${ }_{t_{0}}^{C} D_{t}^{\alpha} e_{3}=-\beta e_{3}+x_{m} e_{2}+y_{s} e_{1}$.

Since the master state trajectory $y_{m}$ remains bounded, i.e., $y_{m} \in$ $\mathcal{L}^{\infty}$, then given that $e_{1} \in \mathcal{L}^{\infty}$, it can be concluded that $y_{s} \in \mathcal{L}^{\infty}$. Since $x_{m} \in \mathcal{L}^{\infty}$ as well, then by using the BIBO stability concepts for fractional systems [28], it can be concluded from (57) that $e_{3} \in \mathcal{L}^{\infty}$, and this concludes the proof.

Lemma 11. (Nonadaptive fractional synchronization using only control signal. $U_{2}$, assuming a known upper bound on parameter $\gamma$.) Let us assume that the parameters $\sigma, \gamma, \beta$ in (8) and (9) are unknown, $\sigma, \gamma, \beta>0$ and $a=1$. It is also assumed that an upper bound $B_{\gamma}$ on the parameter $\gamma$ is known $\left(\gamma<B_{\gamma}\right)$ and that the master system trajectories $x_{m}, y_{m}, z_{m}$ remain bounded. If the following control signals are used in (9)

$$
\begin{aligned}
& U_{1}=-y_{m} z_{s}+y_{s} z_{m}+B_{\gamma \sigma} e_{1} \\
& U_{2}=0 \\
& U_{3}=0
\end{aligned}
$$

then the controlled system (12),(58) is asymptotically stable.

Proof. Using the control signals (58) in (12), the evolution of the synchronization errors results

$$
\begin{aligned}
& { }_{t_{0}}^{C} D_{t}^{\alpha} e_{1}=-\sigma e_{1}+\sigma e_{2} \\
& { }_{t_{0}}^{C} D_{t}^{\alpha} e_{2}=\gamma e_{1}-e_{2}-B_{\gamma} e_{2} \\
& { }_{t_{0}}^{C} D_{t}^{\alpha} e_{3}=-\beta e_{3}+x_{m} e_{2}+y_{s} e_{1} .
\end{aligned}
$$

The stability of the subsystem ${ }_{t_{0}}^{C} D_{t}^{\alpha} e_{1},{ }_{t_{0}}^{C} D_{t}^{\alpha} e_{2}$ can be first analyzed, using the Lyapunov function candidate $V=\frac{1}{2 \sigma} e_{1}^{2}+\frac{1}{2 \gamma} e_{2}^{2}$ and Lemma 1 , obtaining that the fractional derivative of the Lyapunov function is negative definite:

${ }_{t_{0}}^{C} D_{t}^{\alpha} V \leq-\left(e_{1}-e_{2}\right)^{2}+e_{2}^{2}\left(1-\frac{b}{\gamma}\right)$.

Thus using Theorem 2, it can be concluded that $\lim _{t \rightarrow \infty} e_{1}(t)=$ $\lim _{t \rightarrow \infty} e_{2}(t)=0$.

Since $x_{m}, y_{m}, z_{m} \in \mathcal{L}^{\infty}$, the equation for ${ }_{t_{0}}^{c} D_{t}^{\alpha} e_{3}$ in (59) can be analyzed using BIBO stability concepts for fractional systems [28], concluding that $\lim _{t \rightarrow \infty} e_{3}(t)=0$, and this concludes the proof.

Remark 3. Solution using only control signal $U_{3}(t)$ could not be found using the methodology proposed in this paper, which is why this case is not presented in this document.

As a summary of all the results already presented in Section 3, we present in Tables 1 and 2 the main characteristics of the five proposed control strategies for the adaptive case and the nonadaptive case, respectively.

Remark 4. Although the solutions proposed in this paper are for Lorenz systems, the proposed methodology using the fractional extension of Lyapunov direct method and quadratic Lyapunov functions could be applied to achieve synchronization of other type of systems.

\section{Numerical results and simulations}

From the approaches presented in Section 3, it can be concluded analytically that fractional adaptive synchronization of Lorenz systems can be reached, by handling either one or two control signals, using one adjustable parameter. It is also possible to achieve nonadaptive synchronization using one or two control signals, assuming a known upper bound on one or two system parameters.

In the adaptive cases, it can be concluded analytically from the results presented in Section 3 that the controlled system is uniformly stable, although the convergence to zero of the synchronization errors could not be proved analytically, due to a lack of tools to accomplish this task. However, simulations studies have been developed in the context of this research, and they have shown that the synchronization can be effectively achieved in the adaptive cases as well.

This section presents some representative simulation results, for the case when only control signals $U_{2}, U_{3}$ are used, for both the 
Table 1

Summary of the results about fractional adaptive synchronization presented in Section 3.

\begin{tabular}{llllll}
\hline Section & Control signal & $\begin{array}{c}\text { Adjustable } \\
\text { parameter }\end{array}$ & Scale factor & Assumptions & Stability \\
\hline 3.1 & $U_{2}, U_{3}$ & $\theta$ & $a>0$ & $\sigma, \beta>0$ & Uniform stability \\
3.2 & $U_{1}, U_{3}$ & $\theta$ & $a=1$ & $\sigma, \beta>0$ & Uniform stability \\
3.3 & $U_{1}, U_{2}$ & $\theta$ & $a=1$ & $\sigma, \beta>0$ & Uniform stability \\
3.4 & $U_{1}$ & $\theta$ & $a=1$ & $\sigma, \beta>0$ & Uniform stability \\
3.5 & $U_{2}$ & $\theta$ & $a=1$ & $\sigma, \beta>0$ & Uniform stability \\
& & & & $x_{m}, y_{m}, z_{m} \in \mathcal{L}^{\infty}$ & \\
\hline
\end{tabular}

Table 2

Summary of the results about fractional synchronization using upper bounds on some system parameters presented in Section 3.

\begin{tabular}{llllll}
\hline Section & Control signal & $\begin{array}{l}\text { Bounds on } \\
\text { parameters }\end{array}$ & Scale factor & Assumptions & $\begin{array}{l}\text { Stability and } \\
\text { convergence }\end{array}$ \\
\hline 3.1 & $U_{2}, U_{3}$ & $\gamma<B_{\gamma}$ & $a>0$ & $\gamma, \sigma, \beta>0$ & Asymptotic stability \\
3.2 & $U_{1}, U_{3}$ & $\frac{(\gamma+\sigma)^{2}}{4}<B_{\gamma \sigma}$ & $a=1$ & $\beta>0$ & Asymptotic stability \\
3.3 & $U_{1}, U_{2}$ & $\frac{(\gamma+\sigma)^{2}}{4}<B_{\gamma \sigma}$ & $a=1$ & $\beta>0$ & Asymptotic stability \\
3.4 & $U_{1}$ & $\frac{(\gamma+\sigma)^{2}}{4}<B_{\gamma \sigma}$ & $a=1$ & $\beta>0$ & Asymptotic stability \\
3.5 & $U_{2}$ & $\gamma<B_{\gamma}$ & $a=1$ & $\gamma, \sigma, \beta>0$ & Asymptotic stability \\
& & & & $x_{m}, y_{m}, z_{m} \in \mathcal{L}^{\infty}$ & \\
\hline
\end{tabular}

adaptive case (see Subsection 3.1) and the nonadaptive case (see Subsection 3.2). The results presented here illustrate the effectiveness of the proposed synchronization schemes. No simulation results are presented for the rest of the cases for the sake of space, although the conclusions about stability and convergence that can be observed in this case are valid for the rest of the cases as well.

For these simulations, the NInteger Toolbox [29] for Matlab/Simulink was used. The parameter values for the master system (8) and slave system (9), which are assumed to be unknown, are $\sigma=10, \gamma=28$ and $\beta=8 / 3$. It was reported in [30] that for this values, the Lorenz system exhibits a chaotic behavior when the derivation order lies in the interval $0.99 \leq \alpha \leq 1.18$, when $\alpha$ $<0.99$ the Lorenz system tends asymptotically to one of the two attractors and when $\alpha>1.18$ the system exhibits and unstable behavior. Given that the analytical proofs given in this paper are valid in the interval $\alpha \in(0,1)$, only simulations for the cases $\alpha \in[0.99$, 1 ) (chaotic) and $\alpha \in(0,0.99)$ (stable) are presented.

The initial conditions for master and slave systems were chosen as $[-8,-5,6]^{T}$ and $[10,-10,-10]^{T}$, respectively. For this study we took a scale factor $\alpha=1$ as well as $\alpha=2$. In all simulations, the adaptive gain $\delta=1$ was chosen in the adaptive case for simplicity, although the analysis is also valid for any $\delta>0$.

Fig. 1 shows the fractional adaptive case for different values of the derivation order $\alpha$. The norm of the synchronization error vector $e=\left[\begin{array}{lll}e_{1} & e_{2} & e_{3}\end{array}\right]^{T}$ has been plotted in this graphic and a scale factor $a=1$ has been used.

As can be seen, in the three cases the adaptive synchronization is achieved, that is, the norm of the synchronization error converges to zero. It can be noted that the convergence speed is greater as the derivation order $\alpha$ gets closer to 1 . However, the initial overshot is lower as the derivation order $\alpha$ gets farther from 1 , as can be noted in the zoomed part of the graph. This last characteristic is directly related to the control effort, that is, the control effort is lower as the derivation order gets farther from 1.

Fig. 2 shows the evolution of the master and the slave states for two different values of the derivation order, in this case using a scale factor $a=2$.

As can be seen from Fig. 2, the synchronization is effectively achieved in both cases and the use of a scale factor $a=2$ is observed as well. Also it can be noted that when using a derivation order $\alpha=0.5$, the systems exhibit an stable behavior, and when

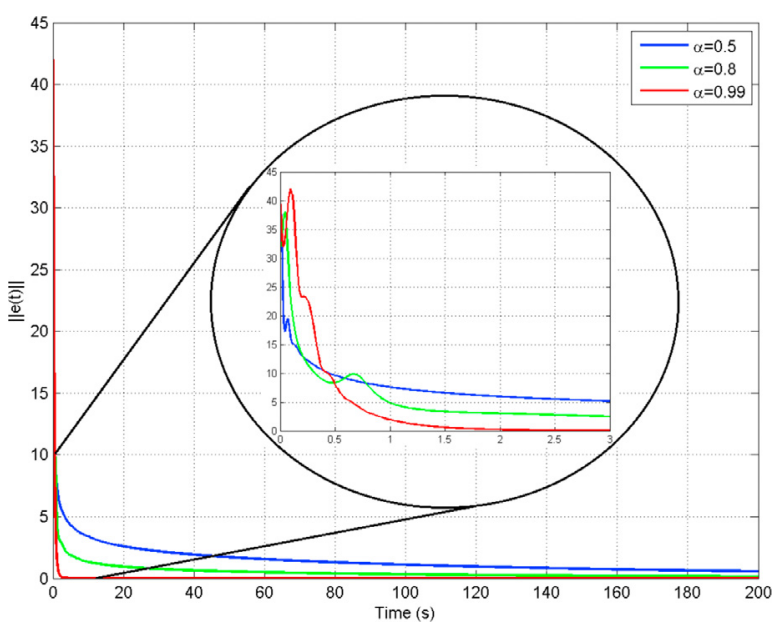

Fig. 1. Evolution of the norm of the synchronization error in the adaptive case, for different values of the derivation order $\alpha$.

$\alpha=0.99$ the systems exhibit a chaotic behavior, as it was found in [30].

Let us now analyze the results in the nonadaptive case. In this simulations the same parameter values and initial conditions than in the adaptive case are used, and the upper bound on the system parameter used is $B_{\gamma}=40$.

Fig. 3 shows the fractional nonadaptive case for different values of the derivation order $\alpha$. The norm of the synchronization error vector $e=\left[\begin{array}{lll}e_{1} & e_{2} & e_{3}\end{array}\right]^{T}$ has been plotted in this graphic as it was done in the adaptive case, and a scale factor $a=1$ has been used.

As can be seen from Fig. 3, the synchronization is achieved for every derivation order used, as it was expected from the stability analysis in Section 3. It is important to note that in this nonadaptive case, the convergence speed does not present important differences if we look at the cases $\alpha=0.5$ and $\alpha=0.8$, as it was in the adaptive case. This is due to the fact that no adaptation process takes place here, so the usual adaptation speed given by the derivation order of the adaptive law does not affect the convergence speed of the errors, as it is in the adaptive case. Of 

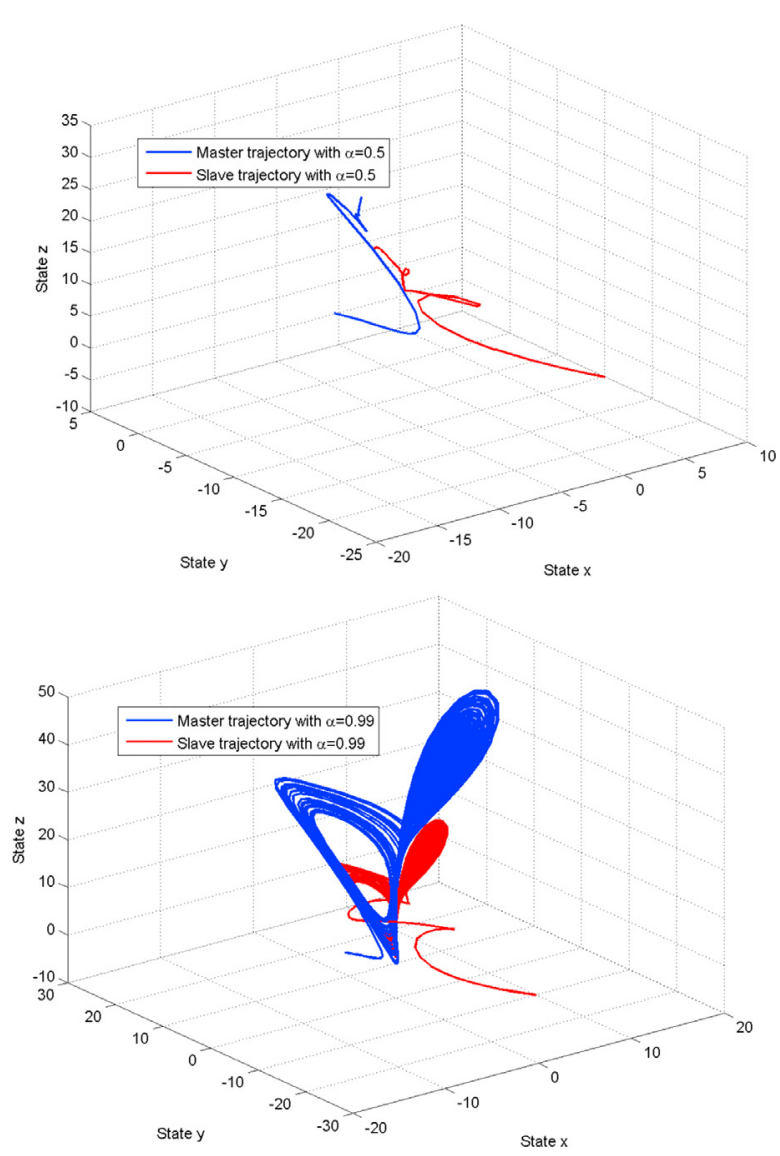

Fig. 2. Evolution of master and slave states for different derivation orders $\alpha$.

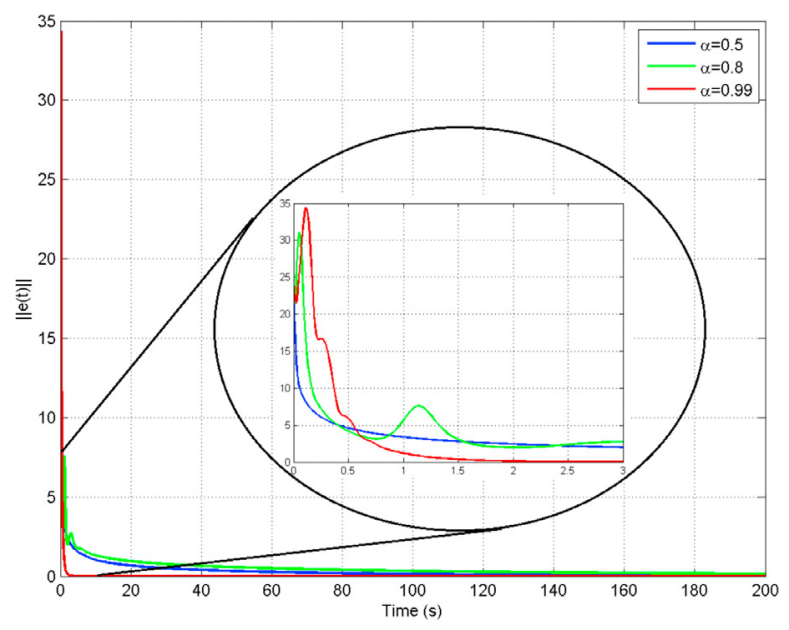

Fig. 3. Evolution of the norm of the synchronization error in the nonadaptive case, for different values of the derivation order $\alpha$.

course, in this case more knowledge of the system is needed in order to construct the control signal than in the adaptive case.

Regarding the transient response in this case, it can be seen from the zoomed part of Fig. 3 that the overshot is higher as the derivation order gets closer to 1 , as it happens in the adaptive case.
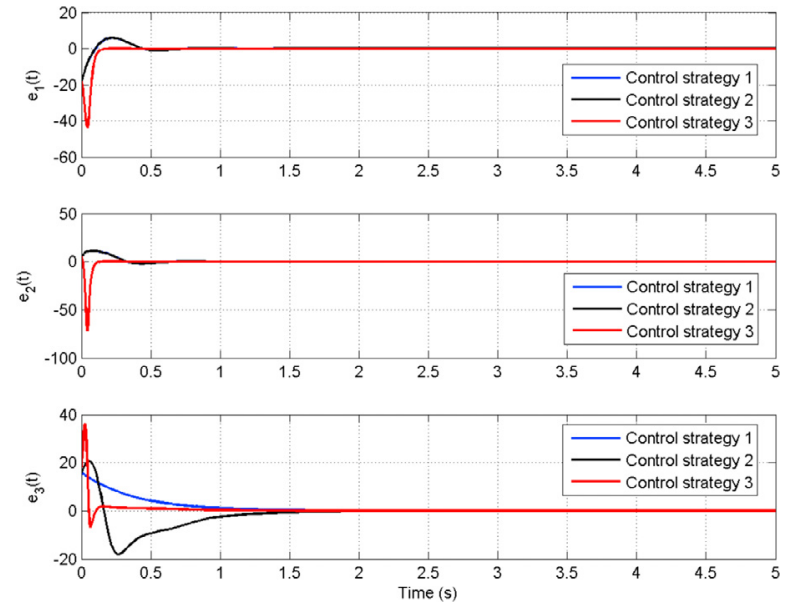

Fig. 4. Evolution of the synchronization errors for three different control strategies.

\subsection{Comparison with another control strategy proposed in the technical literature}

The work presented in this paper has advantage over other techniques, since all the system parameters $\sigma, \gamma, \beta$ are assumed to be unknown, and a reduced number of control signals and adjustable parameters are used to achieve the synchronization.

Although there are no similar works reported in the literature accomplishing all these conditions, we would like to make some comparison between the results reported in this paper and another result reported in literature for the same system, no matter the information needed to implement the control or the number of control signals used. The idea is to analyze the convergence time and the transient response of the synchronization errors for each case, as well as the control effort.

In what follows, we are going to refer to the control strategy proposed in this paper using control signals $U_{2}$ and $U_{3}$ and one adjustable parameter described in Section 3.1 as Control strategy 1. On the other hand, the control strategy proposed in this paper using only control signal $U_{2}$ and one adjustable parameter described in Section 3.5 will be referred as Control strategy 2.

Finally, Control strategy 3 corresponds to the one reported in [6], which does not need any knowledge of the system parameters, but it uses three control signals $U_{1}, U_{2}, U_{3}$ and three adjustable parameters $k_{1}, k_{2}, k_{3}$. Basically, Control strategy 3 uses a feedback control in the form

$$
\begin{aligned}
& U_{1}=k_{1}\left(x_{s}-x_{m}\right) \\
& U_{2}=k_{2}\left(y_{s}-y_{m}\right) \\
& U_{3}=k_{3}\left(z_{s}-z_{m}\right),
\end{aligned}
$$

where the parameters $k_{1}, k_{2}$ and $k_{3}$ are adaptively adjusted using the following adaptive laws:

$$
\begin{aligned}
& \dot{k}_{1}=-\delta e_{1}^{2} \\
& \dot{k}_{2}=-\delta e_{2}^{2} \\
& \dot{k}_{3}=-\delta e_{3}^{2} .
\end{aligned}
$$

The parameter $\delta$ corresponds to the adaptive gain.

Fig. 4 shows the evolution of the synchronization errors using these three control strategies, and Fig. 5 shows the corresponding control signals. In these simulations, the initial conditions for the master and slave systems are the same as in previous simulations, adaptive gains are $\delta=1$ and the initial condition for all the adjustable parameters is 40 . The fractional order used is $\alpha=0.993$ and the simulation time is 5 seconds.

As can be observed in Fig. 4, no big differences can be seen about the convergence time of the synchronization errors between 

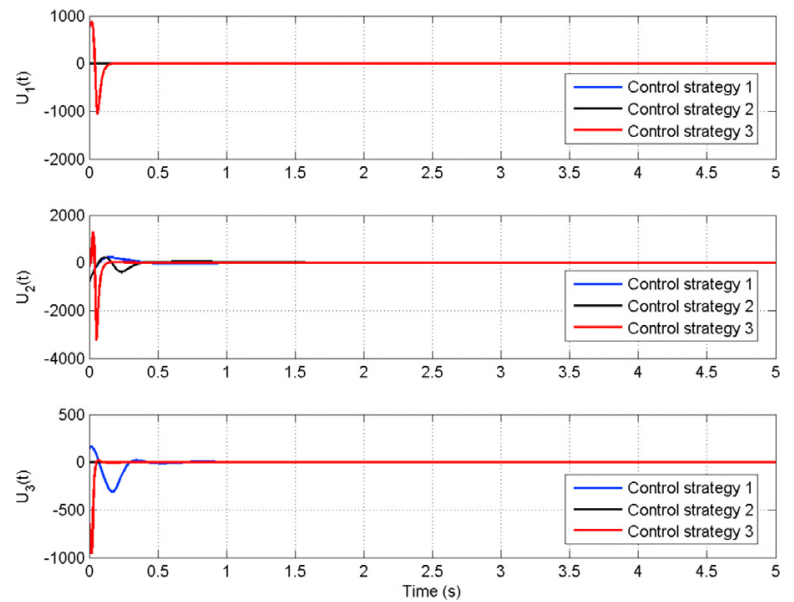

Fig. 5. Evolution of the control signals for three different control strategies.

the three control strategies. In the case of errors $e_{1}$ and $e_{2}$, the difference in the convergence time is about half a second between Control strategy 3 and Control strategies 1 and 2 .

In the case of $e_{3}$, the difference in the convergence time is about 1 second between Control strategy 1 and Control strategy 3. In the case of Control strategy 2 the difference is about 2 seconds with respect to Control strategy 3 and 1 second with respect to Control strategy 1.

Although no big differences can be seen between the three control strategies regarding the convergence time of the synchronization errors, it can be seen from Fig. 4 that the initial overshoot of $e_{1}$ and $e_{2}$ for Control strategy 3 is significantly higher than for Control strategies 1 and 2 . In the case of $e_{3}$, Control strategy 1 does not present overshoot at all, while for Control strategy 2 the magnitude of the overshoot is lesser than for Control strategy 3.

Another aspect of great importance can be seen in Fig. 5, where it can be observed that the initial control effort is particularly high for Control strategy 3, compared to Control strategies 1 and 2 . Since Control strategy 2 uses only control signal $U_{2}$ and Control strategy 1 uses only control signals $U_{2}$ and $U_{3}$, this behavior represents a great advantage of using control strategies proposed in this paper.

We must point out that Control strategy 1 and 2 use not only less control signals ( 2 and 1 respectively) than Control strategy 3 , but they also use only one adjustable parameter, compared to three used in Control strategy 3.

Of course, the simulations presented here were carried out using specific values for adaptive gains, initial conditions of the estimated parameters, order of the fractional adaptive laws in the case of Control strategies 1 and 2, among others. Thus, the results could be different under different values of all these parameters. An optimization procedure could be a great option to find specific parameters (adaptive gains, fractional orders, initial conditions) that guarantee desired results.

\section{Conclusions}

In this paper, the analysis of the adaptive synchronization of two fractional Lorenz systems has been presented, as well as the analysis of nonadaptive synchronization. The synchronization was studied based on theoretical results and complemented by simulations, analyzing the behavior of the synchronization errors.

The study performed in this paper indicates that fractional adaptive synchronization of Lorenz systems can be achieved with a reduced number of parameters and signals, under mild assumptions. This can be done by using two control signals and one ad- justable parameter, or even using one control signal and one adjustable parameter. The study also indicates that nonadaptive synchronization can be achieved under the same mild assumptions, using one or two control signals and upper bounds on one or two of the system unknown parameters.

In both solutions, adaptive and nonadaptive, the stability of the resulting schemes was analytically proved, using the fractional extension of Lyapunov direct method. The convergence to zero of the synchronization errors was proved in the nonadaptive case, using the fractional extension of the Lyapunov direct method as well. In the adaptive case, however, the convergence to zero of the synchronization errors could not be analytically proved, due to a lack of available tools to accomplish this task, being this topic currently under investigation. Nevertheless, simulation studies indicate that the synchronization errors do converge to zero in the adaptive case too.

\section{Acknowledgments}

The results reported in this paper have been financed by CONICYT-Chile, under the Basal Financing Program FB0809 "Advanced Mining Technology Center," FONDECYT Project 1150488, "Fractional Error Models in Adaptive Control and Applications" and FONDECYT 3150007, "Postdoctoral Program 2015."

\section{References}

[1] Agrawal S, Das S. Projective synchronization between different fractional-order hyperchaotic systems with uncertain parameters using proposed modified adaptive projective synchronization technique. Math Methods Appl Sci 2014;37:2164-76.

[2] Blasius B, Huppert A, Stone L. Complex dynamics and phase synchronization in spatially extended ecological system. Nature 1999;399:354-9.

[3] Freeman W. Characteristics of the synchronization of brain activity imposed by finite conduction velocities of axons. Int J Bifurcat Chaos 2000;10:26307-22.

[4] Zhou P, Yang X. A novel hybrid function projective synchronization between different fractional-order chaotic systems. Discrete Dyn Nat Soc 2011;2011:1-15.

[5] Feng M, Wang X, Wei Q. Adaptive robust synchronization of fractional-order chaotic system with disturbance. J Vib Control 2015;21:2259-65.

[6] Leung A, Li X, Chu Y, Rao X. Synchronization of fractional-order chaotic systems using unidirectional adaptive full-state linear error feedback coupling. Nonlinear Dynam 2015;82:185-99.

[7] Li R, Chen W. Lyapunov-based fractional-order controller design to synchronize a class of fractional-order chaotic systems. Nonlinear Dynam 2014;76:785-95.

[8] Yang L, Jiang J. Adaptive synchronization of drive-response fractional-order complex dynamical networks with uncertain parameters. Commun Nonlinear Sci Numer Simul 2014;19:1496-506.

[9] Zhang R, Yang S. Robust synchronization of two different fractional-order chaotic systems with unknown parameters using adaptive sliding mode approach. Nonlinear Dynam 2013;71:269-78.

[10] Chen L, Wu R, He Y, Chai Y. Adaptive sliding-mode control for fractional-order uncertain linear systems with nonlinear disturbances. Nonlinear Dynam 2015;80:51-8.

[11] Chen D, Zhao W, Liu X, Ma X. Synchronization and antisynchronization of a class of chaotic systems with nonidentical orders and uncertain parameters. J Comput Nonlinear Dynam 2015;10:1-8.

[12] Wang Q, Ding D, Qi D. Mittag-leffler synchronization of fractional-order uncertain chaotic systems. Chinese Phys B 2015;24:1-6.

[13] Boulkroune A. Bouzeriba A. Bouden T. Fuzzy generalized projective synchronization of incommensurate fractional-order chaotic systems. Neurocomputing 2016;173:606-14.

[14] Zhou P, Bai R. One adaptive synchronization approach for fractional-order chaotic system with fractional-order $1<\mathrm{q}<2$. Sci World J 2014;2014:1-7.

[15] Yuan J, Shi B, Ji W. Adaptive sliding mode control of a novel class of fractional chaotic systems. Adv Math Phys 2013;2013:1-14.

[16] Gao L, Wang Z, Zhou K, Zhu W, Wu Z, Ma T. Modified sliding mode synchronization of typical three-dimensional fractional-order chaotic systems. Neurocomputing 2015;166:53-8.

[17] Yin C, Cheng Y, Chen Y, Stark B, Zhong S. Adaptive fractional-order switchingtype control method design for 3d fractional-order nonlinear systems. Nonlinear Dynam 2015;82:39-52.

[18] Kilbas A, Srivastava H, Trujillo J. Theory and applications of fractional differential equations. Amsterdam: Elsevier; 2006.

[19] Matignon D. Stability results for fractional differential equations with applications to control processing. In: Multiconference on Computational Engineering in Systems Applications. Lille, France: GERF, Ecole Centrale de Lille; 1996. p. 963-8. 
[20] Duarte-Mermoud MA, Aguila-Camacho N, Gallegos JA, Castro-Linares R. Using general quadratic lyapunov functions to prove lyapunov uniform stability for fractional order systems. Commun Nonlinear Sci Numer Simul 2015;22:650-9.

[21] Li Y, Chen Y, Podlubny I. Stability of fractional-order nonlinear dynamic systems: Lyapunov direct method and generalized mittag leffler stability. Comput Math Appl 2010;59:1810-21.

[22] Aguila-Camacho N, Duarte-Mermoud MA, Gallegos JA. Lyapunov functions for fractional order systems. Commun Nonlinear Sci Numer Simul 2014;19:2951-7.

[23] Alikhanov A. A priori estimates for solutions of boundary value problems for fractional-order equations. Differ Equations 2010:46:660-6.

[24] Lorenz E. Deterministic non-periodic flow. J Atmos Sci 1963;20:130-41.

[25] Wang Y, Guan Z. Generalized synchronization of continuous chaotic system. Chaos Soliton Fract 2006;27:97-101.
[26] Delgado-Aguilera E, Duarte-Mermoud MA, Aguila-Camacho N. Minimal adaptive synchronization of lorenz systems. IMA J Math Control Inform 2015 [submitted].

[27] Narendra KS, Annaswamy AM. Stable adaptive systems. Mineola, New York: Dover Publications Inc.; 2005.

[28] Matignon D. Stability properties for generalized fractional differential systems. ESAIM: Proc Fract Differ Syst: Models Methods Appl 1998;5:145-58.

[29] Valerio D, Da Costa JS. Ninteger: a non-integer control toolbox for matlab. In: Fractional derivatives and applications. Bordeaux, France: IFAC; 2004.

[30] Delgado-Aguilera E. Adaptive synchronization of fractional order systems of the lorenz type [Ms.c. thesis], Santiago, Chile: Electrical Engineering Department, University of Chile; 2012. [in spanish]. 